\title{
Correlación entre los aspectos personales y laborales con el desgaste profesional de enfermería en un hospital de segundo nivel
}

Nieto-Martínez Elvia*, Hernández- Castañón Ma. Alejandra**, Garza-González Beatriz**, Hernández-Rodríguez Verónica Margarita**, Sánchez-Perales Mercedes**

\section{- Resumen}

- Introducción: el desgaste profesional de enfermería (DPE) es un

- problema de salud laboral de las organizaciones, especialmente las

- de salud. El de enfermería es un grupo vulnerable, pues requiere el

- despliegue de actividades que necesitan control mental y emocio-

- nal por el manejo de pacientes en los centros de trabajo. Objetivo:

- determinar la relación de los aspectos personales y laborales con

- el desgaste profesional de enfermería de un hospital de segundo

- nivel de atención. Metodología: estudio transversal correlacional en

- 165 enfermeras por muestreo probabilístico de un total de 227. Se

- aplicó el Cuestionario Desgaste Profesional de Enfermería (CDPE-

- A), con 65 preguntas enfocadas a datos personales y laborales, y a

- las cuatro dimensiones del DPE:Antecedentes, Síndrome, Personali-

- dadlafrontamiento y Consecuencias, con Alfa de Cronbach de 0.88 .

- El DPE se clasificó como bajo, medio o alto. Se usó la estadística

- descriptiva, así como la correlación de Pearson y Chi cuadrada con

- un nivel de confianza del 95\%. El estudio fue de riesgo mínimo.

- Resultados: el $90.1 \%$ de la muestra fue del sexo femenino, con un

- promedio de edad de $42 \pm 8.89$ años y el $46.3 \%$ manifestó contar

- con licenciatura. El 34.6\% laboraba en el turno nocturno, el prome-

- dio de antigüedad fue de $16 \pm 7.74$ años, el de las horas de trabajo

- fue de $42 \pm 13$ y el del número de pacientes fue de $7 \pm 13$. El $84 \%$

- presentaron nivel medio de DPE. La edad y la antigüedad se rela-

- cionaron con la dimensión personalidad/afrontamiento y el número

- de horas de trabajo con la de antecedentes. Conclusión: Ocho de

- cada diez enfermeras presentaron nivel medio de DPE, por lo que

- es pertinente que se generen programas de prevención, promoción

- e intervención en corresponsabilidad tanto de la institución como

- del propio personal, para reducir esta problemática. LUXMÉDICA

- AÑO 15, NÚMERO 44, MAYO-AGOSTO 2020, PP 59-70.

- Palabras clave: desgaste profesional, enfermería, salud laboral.

\section{Abstract}

Introduction: Nursing burnout (NB) is an occupational health problem, especially in health organizations. Nursing is a vulnerable group since it involves activities that need mental and emotional control for the management of patients in the workplace. Objective: To determine the relationship between personal and work aspects with nursing burnout in a second-level hospital. Methods: This is a cross-sectional study of 165 nurses by a probabilistic sampling of a total of 227. The Nursing Burnout Questionnaire (NBQ) was applied, with 65 questions focused on personal and work data, and the four dimensions of NB, background, syndrome, personalityl coping, and consequences; it had a Cronbach's alpha of 0.88. NB was classified as low, moderate, or high. Descriptive statistics were used, as well as the Pearson and Chi-square correlation with a 95\% confidence level. The study had minimal risk. Results: $90.1 \%$ were female with an average age of $42 \pm 8.89$ years and $46.3 \%$ had a bachelor's degree; $34.6 \%$ worked in the night shift for an average of $16 \pm 7.74$ years, working $42 \pm 13$ hours, with $7 \pm 13$ patients; $84 \%$ presented a moderate level of NB. Age and number of years working were related to the personality/coping dimension and the number of hours of work with the background. Conclusions: Eight out of ten nurses presented a moderate level of NB; therefore, it is pertinent that prevention, promotion, and intervention programs be generated in co-responsibility of both the institution and the staff to reduce this problem. LUXMÉDICA AÑO 15, NÚMERO 44, MAYOAGOSTO 2020, PP 59-70.

* Nieto-Martínez Elvia. Licenciada en Enfermería, adscrita al Hospital General de Querétaro. Correo electrónico cri.el@hotmail.com

** Hernández- Castañón Ma. Alejandra. Profesora Investigadora de la Universidad Autónoma de Querétaro. Correo electrónico alehdez983@yahoo. com.mx

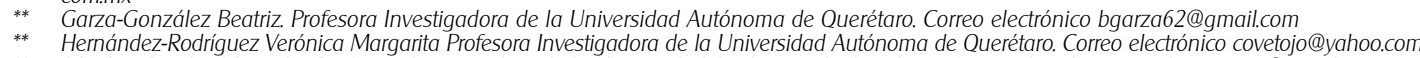

** Sánchez-Perales Mercedes Profesora Investigadora de la Universidad Autónoma de Querétaro. Correo electrónico sanchezpe.msp@gmail.com

Fecha de recibido 28 de mayo 2019

Fecha de aceptación: 27 de septiembre 2019

Correspondencia: LE Elvia Nieto Martínez. Adscripción de trabajo: Hospital General de Querétaro. Domicilio: Avenida 5 de febrero, No.101, Colonia Los Virreyes, Santiago de Querétaro, Qro. México. Teléfono (442) 101 2900. Código Postal: 76170. Correo electrónico: cri.el@hotmail.com. 


\section{Introducción}

En los últimos veinte años ha habido una creciente preocupación por los efectos del estrés sobre los profesionales de enfermería, ya que sus condiciones de salud y trabajo se han deteriorado progresivamente en todo el mundo, situación que se ha visto reflejada en la disminución de la demanda del ingreso a la carrera y a la deserción en la profesión. ${ }^{1}$

Por tal motivo, la Organización Internacional del Trabajo (OIT), la Organización Mundial de la Salud (OMS) y la American Nurses Association (ANA), refieren preocupación por el personal de enfermería en relación con su salud y seguridad en el entorno laboral; en ese sentido, la OIT expidió desde 1977 el convenio 149, Sobre empleo, condiciones de vida y de trabajo del personal de enfermería, y la OMS en su asamblea mundial realizada en mayo de 1922, expidió la resolución WHA 42.27, donde recomienda a los países desarrollar estrategias específicas para el fortalecimiento de la salud y seguridad en el entorno laboral. 1,2

La Organización Panamericana de la Salud (OPS) considera el lugar de trabajo como un entorno prioritario para la promoción de la salud en el siglo XXI, esencial no sólo para lograr la salud de los trabajadores sino también para hacer un aporte positivo a la productividad, la motivación laboral, el espíritu y la satisfacción en el trabajo, así como la calidad de vida en general. ${ }^{2}$

Dichas organizaciones mencionan que las condiciones del trabajo de enfermería implican exposición al dolor, muerte y conflictos interpersonales. La respuesta individual a estas situaciones puede ser de dos tipos: a) psicológica, causando ansiedad, irritación y depresión; y b) psicosomática, manifestada por dolores de cabeza, náuseas, y trastornos del sueño, pudiendo tener un impacto negativo en la seguridad del paciente y en la calidad de los cuidados prestados. Por lo anterior, es importante cuidar la salud del profesional de enfermería, ya que es un grupo vulnerable para padecer el Síndrome de Burnout (SB) o Desgaste Profesional. ${ }^{1,3,4}$

En el año 2005, la Asamblea Mundial de la Salud estableció líneas de acción encaminadas a la seguridad del paciente al grado de llevarlo a una obligatoriedad dentro de los programas de la administración pública federal, mencionándose las siguientes estrategias: "4.3 Integrar sectorialmente las acciones de prevención de enfermedades; 8.3 Promover la productividad laboral mediante entornos de trabajos saludables, a través de la instrumentación de medidas para reducir los riesgos en el trabajo y promover entre los trabajadores estilos de vida saludables". 3

Sin embargo, no se cumplen dichas estrategias ya que el profesional de enfermería sigue enfermándose, con el consiguiente ausentismo laboral, lo cual vulnera al individuo en su carácter de 
trabajador, a la colectividad de la institución, la calidad de los servicios de salud y al cumplimiento de los objetivos de la misma organización. ${ }^{2,3}$

En este contexto, se puede mencionar lo que plantea Maslach citado por Díaz ${ }^{5}$, con relación a que el Síndrome de Bournout es el resultado de una respuesta prolongada a los estresores interpersonales crónicos en el trabajo, de la estructura y el funcionamiento del lugar del trabajo, que limita la forma en que la persona interactúa y realiza su labor. ${ }^{5}$

A nivel nacional e internacional, la prevalencia del síndrome de Burnout se presenta como consecuencia de los fuertes cambios que se produjeron desde la década de los años 70 en el mundo laboral de los países altamente industrializados y el desplazamiento de los riesgos laborales del campo físico al campo mental, lo que generó un aumento vertiginoso de casos de Burnout en Europa entre el año 1990 al 2010. En México la prevalencia de Burnout fue de $74.6 \%$ en el año 2014, el aumento se debe a la situación por la cual atraviesa el sistema de salud en México afectando el salario, incentivos y estabilidad laboral. 5,6

Lazarus y Folkman ${ }^{6}$ mencionan que es sumamente importante el ambiente laboral en el que se desempeñan los profesionales de enfermería, ya que el estrés es el resultado de la relación individuo con su entorno laboral, aunado al hecho de que este personal es predominantemente femenino, por arriba de la media internacional y nacional afectando la calidad de vida de las enfermeras, ${ }^{2,6}$ al asumir una doble función (trabajo remunerado y el doméstico). Como señala Balseiro, ${ }^{2}$ esto ha significado una sobrecarga de trabajo y aumento de los niveles de estrés, ansiedad y depresión, fenómenos que evidencian los efectos negativos del trabajo y del hogar sobre la salud mental de la mujer trabajadora.

Las consecuencias de estos riesgos psicosociales y laborales afectan de manera directa a la persona, generando problemas de tipo psicológico, fisiológico y económico; y a quien le proporciona sus cuidados, así como a la organización al aumentar el ausentismo, menor capacidad del trabajo, frecuentes cambios de servicios, conflictos interpersonales, hipercrítica a los compañeros, insatisfacción en el trabajo, bajos sueldos y turnos nocturnos, ${ }^{2,7}$ elementos propicios para el desarrollo de una enfermedad profesional o laboral.

Para esta investigación se aplicaron los planteamientos de Moreno-Jiménez y cols. $(2000)^{8}$ relacionados al desgaste profesional y que se fundamentan en un modelo teórico transaccional e Interactivo, que considera la interacción del desgaste profesional producto del contexto laboral de las características individuales y del tipo de afrontamiento adoptado, y se basa en cuatro dimensiones que se describen en la figura 1. 


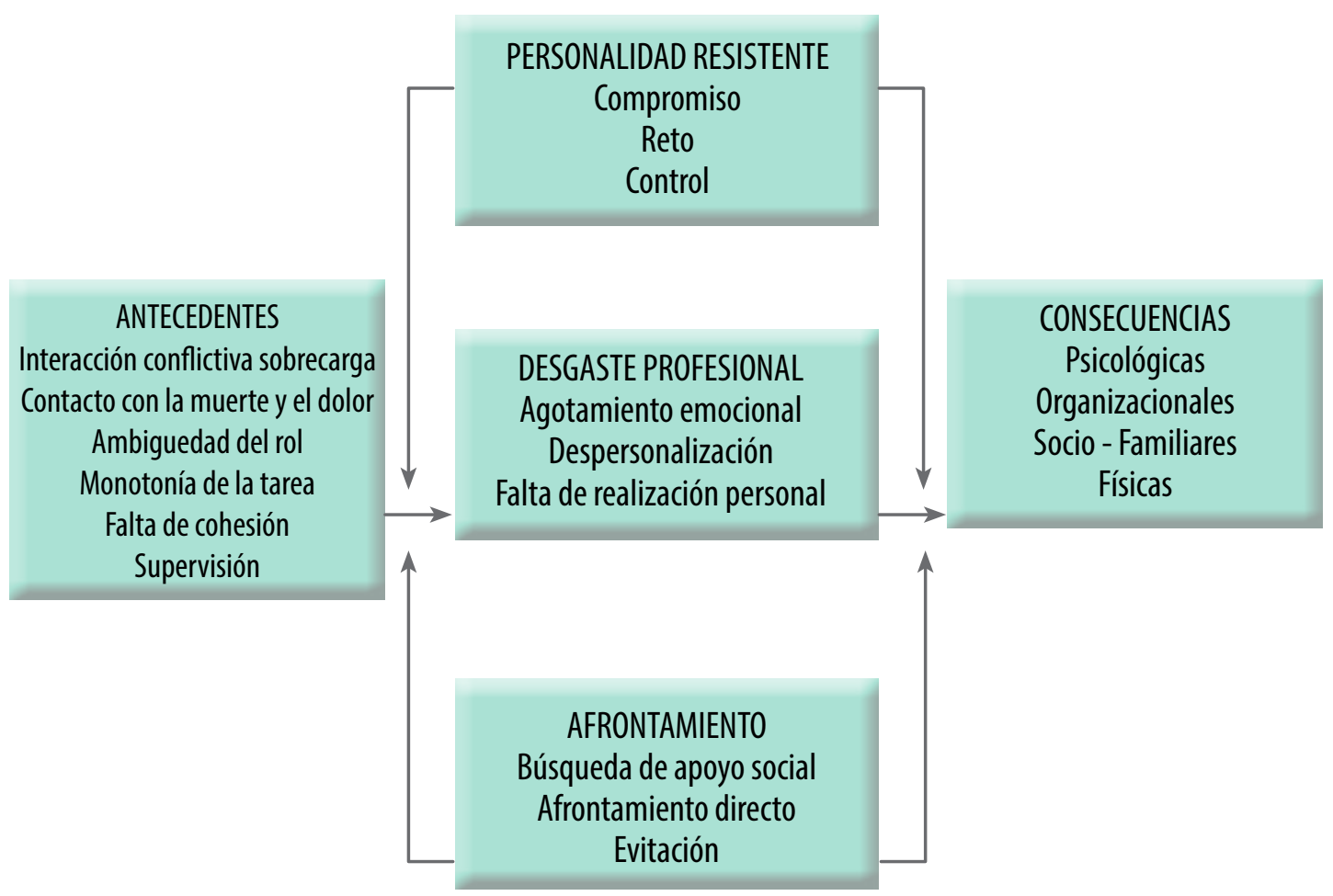

Figura 1. Diagrama representativo del marco teórico del CDPE (Moreno-Jiménez 2000).

A partir de la problemática antes mencionada y de las repercusiones que implica el desgaste profesional en el gremio de enfermería, se realizó esta investigación, con el propósito de determinar la relación entre los aspectos personales y laborales con el desgaste profesional de enfermería en un hospital de segundo nivel de atención en la ciudad de Querétaro, de tal manera que los hallazgos fundamenten la instrumentación de estrategias organizacionales y preventivas futuras en el sector salud.

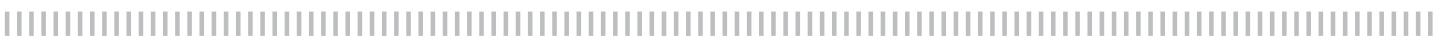

\section{Material y métodos}

Estudio observacional, transversal y correlacional en personal de enfermería, de base (federal y estatal) y regularizados hasta el 2012, adscritos a un hospital público de segundo nivel de atención de la ciudad de Querétaro, Qro. La población total fue de 227 y se obtuvo una muestra probabilística estratificada por turno de 165 enfermeras. La muestra final fue de 162 enfermeras debido a que tres cuestionarios fueron llenados de manera incompleta.
Se aplicó el Cuestionario de Desgaste Profesional de Enfermería (CDPE-A) de Moreno-Jiménez y cols. (2000) en su versión abreviada de 65 preguntas. Cabe destacar que, en el año 2000, los autores elaboraron la versión larga con 174 preguntas cuya confiabilidad, según evidencia científica, oscila en un Alfa de Cronbach de .57 a .94 con una media de .81. En la presente investigación, se obtuvo una Alfa de Cronbach de .88. En la literatura se localizaron investigaciones que han aplicado el CDPE-A en países como 
España, Colombia y México. En este último también se aplicó en profesionales médicos, y reportaron confiabilidad similar a la del autor del instrumento..$^{8-12}$

Las variables que mide el cuestionario son aspectos personales (sexo, edad, número de hijos, relaciones personales, nivel de estudios) y laborales (tipo de contratación, servicio donde labora, número de paciente por jornada, interacción con pacientes (atención directa, tiempo en el servicio, puesto, turno, antigüedad y horas de trabajo por semana) del personal de enfermería, así como la variable desgaste profesional a través de cuatro dimensiones: a) antecedentes, que incluye 16 preguntas sobre ambigüedad del rol, contacto con el dolor y la muerte, interacción conflictiva y sobrecarga; b) síndrome con 12 preguntas enfocadas en cansancio, despersonalización y falta de realización; c) personalidad y afrontamiento con 21 reactivos sobre compromiso, reto, control, apoyo social, evitación y afrontamiento y d) consecuencias, conformada por 16 preguntas de consecuencias psíquicas, profesionales, sociofamiliares y física.
Las preguntas se miden en una escala tipo Likert de cuatro puntos que van del uno totalmente en desacuerdo hasta el cuatro totalmente de acuerdo. Se interpreta con los puntos de corte entre 1 y 4 . El nivel bajo $1>\mathrm{y}<2$, nivel medio $2>\mathrm{y}$ $<3$ y nivel alto $3>$ y $<4$. El procesamiento de los datos se realizó en el IBM SPSS Statistics v.25. Para las variables cualitativas se calcularon frecuencias y porcentajes y para las cuantitativas media y desviación estándar. Se aplicaron las pruebas de correlación de Pearson ( $r$ y y la Chi-Cuadrada (X2) para relacionar los aspectos personales y laborales con las dimensiones del desgaste profesional (CDPE) con un nivel de confianza del $95 \%$.

De acuerdo con el Reglamento de la Ley General de Salud en Materia de Investigación es un estudio de riesgo mínimo (Art. 17) y se garantiza la autonomía y confidencialidad de los participantes con base en el Artículo 14, apartado V, VI, VII. ${ }^{14}$ El estudio fue aprobado por el Comité de Investigación del Hospital General de Querétaro y por la Facultad de Enfermería de la Universidad Autónoma de Querétaro.

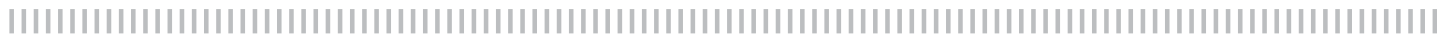

\section{Resultados}

\section{Características sociodemográficas y per- sonales de los participantes}

La muestra final de personal de enfermería fue de 162 , de los cuales $90.1 \%$ fue de sexo femenino, el $41.9 \%$ se encontró entre los 37 y 46 años con una media de $42 \pm 8.89$ años. Más del $50 \%$ refirió tener pareja habitual y $24.7 \%$ sin pareja. Con relación a si tienen hijos, el $75.9 \%$ respondió afirmativamente con una media de $1 \pm .5$. El nivel académico con mayor porcentaje fue el de licenciatura seguido del técnico, con $46.3 \%$ y $43.2 \%$ respectivamente.

\section{Características laborales del personal de enfermería}

El $69.8 \%$ del personal de enfermería contó con base federal, laborando principalmente en los servicios de cirugía con $21.6 \%$; urgencias, $14.8 \%$; medicina interna $y$ UCIA cada una con $13.6 \%$. Predominó el personal operativo con el $89.5 \%$ y laboró en su mayoría en los turnos nocturno y matutino el $34.6 \%$ y el $30.2 \%$ respectivamente, cabe destacar que esta distribución fue proporcional al número de personal por turno. El $53.7 \%$ de las enfermeras tuvo una antigüedad entre 11 y 20 años de laborar en la institución, con una media de 16士7.74 años. 
Dentro de las características laborales es importante conocer el tiempo que dedica el personal a sus funciones, así en la tabla 1 se muestra que el $41.9 \%$ del personal refirió trabajar entre 24 y 36 horas por semana con una media de $42 \pm 13$ ho- ras; además, se observó que, en su jornada laboral, el $43.9 \%$ atiende entre 5 y 9 pacientes con un promedio de $7 \pm 13$. Por otra parte, el $79 \%$ dedicó más del $60 \%$ a la interacción directa con el paciente principalmente en los cuidados.

\section{Tabla I}

\section{Características laborales del personal de enfermería participante en el estudio. (n=162)}

\begin{tabular}{|c|c|c|}
\hline Características & $f$ & $\%$ \\
\hline \multicolumn{3}{|c|}{ Número de horas trabajadas semanales } \\
\hline 24 a 36 & 68 & 41.9 \\
\hline 37 a 49 & 62 & 38.2 \\
\hline Más de 50 & 32 & 19.6 \\
\hline \multicolumn{3}{|c|}{ Cantidad de pacientes } \\
\hline$<4$ & 63 & 38.8 \\
\hline 5 a 9 & 71 & 43.8 \\
\hline 10 a 14 & 18 & 29 \\
\hline$>15$ & 10 & 6.1 \\
\hline \multicolumn{3}{|c|}{ Interacción con los pacientes } \\
\hline Más del $60 \%$ & 128 & 79 \\
\hline 41 a $60 \%$ & 22 & 13.6 \\
\hline 20 y $40 \%$ & 5 & 3.1 \\
\hline Menos del $20 \%$ & 7 & 4.3 \\
\hline
\end{tabular}

Fuente: CDPE-A

\section{Desgaste Profesional de Enfermería}

En relación con el Desgaste Profesional de Enfermería (CDPE), los resultados en cada dimensión se presentan en la figura 2 , en donde se advierte que el personal de enfermería, en tres de las dimensiones, se ubicaron en el nivel medio de desgaste profesional con porcentajes que oscilaron entre el $72.2 \%$ y el $84 \%$. En la dimensión consecuencias, que involucra aspectos como psíquicos, profesionales, sociofamiliares y físicos, el $22.2 \%$ del personal tuvo un nivel alto de degaste profesional.

Figura 2. Nivel de desgaste profesional de enfermería en las cuatro dimensiones de estudio ( $n=162)$.

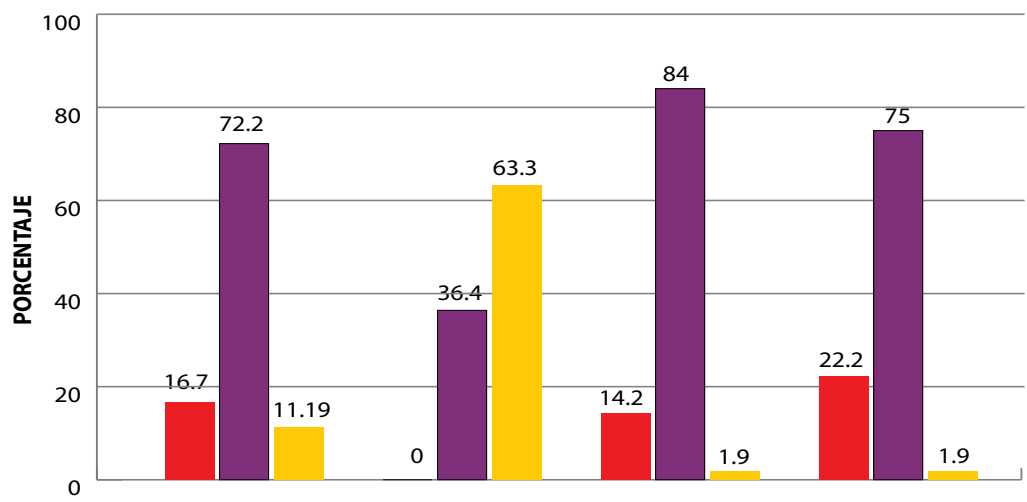

Nivel alto

Nivel medio

Nivel bajo

Fuente: CDPE-A 
De acuerdo con el resultado global del Desgaste Profesional que incluye los resultados de las cuatro dimensiones, se encontró que en el personal de enferme- ría de esta investigación prevalece el nivel medio de desgaste representado por el $84 \%$ (ver figura 3 ).

Figura 3. Desgaste profesional global del personal de enfermería. $(n=162)$

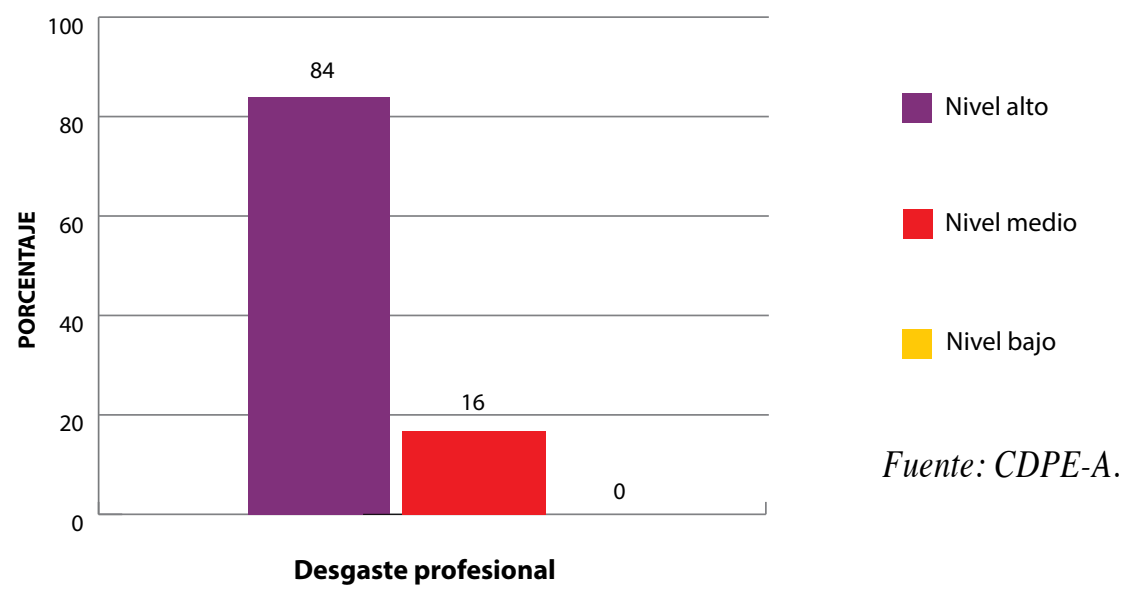

\section{Relación del desgaste profesional de enfermería con los aspectos personales y laborales}

Se procedió a aplicar la prueba de correlación de Pearson ( $r$ ) para las variables escalares, puntaje global de Desgaste Profesional de Enfermería y los aspectos personales como edad, antigüedad, tiempo en el servicio, número de pacientes que atiende y horas de trabajo a la semana; en ninguna de las variables se obtuvo relación estadísticamente significativa. Sin embargo, al correlacionar las dimensiones antecedentes, síndrome, personalidad/ afrontamiento y consecuencias, la edad y antigüedad laboral mostraron correlación positiva baja $r=.246, p=.002$ y $r=.321$, $p=.000$, respectivamente con la dimen- sión de antecedentes, como se presenta en la tabla 2. Por otra parte, las horas de trabajo por semana mostraron una correlación positiva baja $(r=.210, p=.007)$ con la dimensión consecuencias, resultado que se encuentra en la tabla 3.

Los hallazgos anteriores permiten inferir que conforme avanza la edad y los años laborales, se tienen más estrategias de personalidad y afrontamiento por lo que disminuye la posibilidad de tener desgaste profesional. También se puede afirmar que si se incrementan las horas de trabajo se elevan las consecuencias del desgaste profesional en enfermería. 


\section{Tabla 2}

Correlaciones de Pearson (r) para la dimensión personalidad/afrontamiento del Desgaste Profesional de Enfermería y los aspectos personales y laborales.

\begin{tabular}{|c|c|c|c|c|c|c|c|}
\hline Aspectos/ & Dimensión & $\begin{array}{l}\text { Edad en } \\
\text { años }\end{array}$ & $\begin{array}{c}\text { Años de } \\
\text { antigüedad }\end{array}$ & $\begin{array}{c}\text { Tiempo } \\
\text { en el } \\
\text { servicio }\end{array}$ & $\begin{array}{l}\text { Número de } \\
\text { pacientes } \\
\text { por turno }\end{array}$ & $\begin{array}{c}\text { Horas } \\
\text { por } \\
\text { semana }\end{array}$ & $\begin{array}{l}\text { Dimensión per- } \\
\text { sonalidad y } \\
\text { afrontamiento }\end{array}$ \\
\hline \multirow[t]{2}{*}{$\begin{array}{l}\text { Edad en } \\
\text { años }\end{array}$} & $\begin{array}{c}\text { Correlación } \\
\text { Pearson }\end{array}$ & 1 & & & & & \\
\hline & $\begin{array}{c}\text { Significancia } \\
\text { unilateral }\end{array}$ & & & & & & \\
\hline \multirow[t]{2}{*}{$\begin{array}{c}\text { Años de } \\
\text { antigüedad }\end{array}$} & $\begin{array}{c}\text { Correlación } \\
\text { Pearson }\end{array}$ & $.666^{* *}$ & 1 & & & & \\
\hline & $\begin{array}{c}\text { Significancia } \\
\text { unilateral }\end{array}$ & .000 & & & & & \\
\hline \multirow[t]{2}{*}{$\begin{array}{l}\text { Tiempo en el } \\
\text { servicio }\end{array}$} & $\begin{array}{c}\text { Correlación } \\
\text { Pearson }\end{array}$ & $.224^{* *}$ & $.204^{* *}$ & 1 & & & \\
\hline & $\begin{array}{c}\text { Significancia } \\
\text { unilateral }\end{array}$ & .004 & .009 & & & & \\
\hline \multirow[t]{2}{*}{$\begin{array}{l}\text { Número de } \\
\text { pacientes } \\
\text { por turno }\end{array}$} & $\begin{array}{c}\text { Correlación } \\
\text { Pearson }\end{array}$ & .079 & .122 & .060 & 1 & & \\
\hline & $\begin{array}{c}\text { Significancia } \\
\text { unilateral }\end{array}$ & .316 & .123 & .449 & & & \\
\hline \multirow[t]{2}{*}{$\begin{array}{c}\text { Horas por } \\
\text { semana }\end{array}$} & $\begin{array}{c}\text { Correlación } \\
\text { Pearson }\end{array}$ & $-.180^{*}$ & -.098 & $-.164^{*}$ & -.020 & 1 & \\
\hline & $\begin{array}{c}\text { Significancia } \\
\text { unilateral }\end{array}$ & .022 & .216 & .037 & .797 & & \\
\hline \multirow{2}{*}{$\begin{array}{l}\text { Dimensión } \\
\text { personalidad } \\
\text { y afronta- } \\
\text { miento }\end{array}$} & $\begin{array}{c}\text { Correlación } \\
\text { Pearson }\end{array}$ & $.246^{* *}$ & $.321^{* *}$ & .149 & .004 & -.021 & 1 \\
\hline & $\begin{array}{c}\text { Significancia } \\
\text { unilateral }\end{array}$ & .002 & .000 & .058 & .956 & .788 & \\
\hline
\end{tabular}

**Correlación significativa en un nivel 0.01 (unilateral) 


\section{Tabla 3}

Correlaciones de Pearson (r) para la dimensión consecuencias del Desgaste Profesional de Enfermería y los aspectos personales y laborales.

\begin{tabular}{|c|c|c|c|c|c|c|c|}
\hline Aspectos/ & Dimensión & $\begin{array}{l}\text { Edad en } \\
\text { años }\end{array}$ & $\begin{array}{c}\text { Años de } \\
\text { antigüedad }\end{array}$ & $\begin{array}{c}\text { Tiempo } \\
\text { en el } \\
\text { servicio }\end{array}$ & $\begin{array}{l}\text { Número de } \\
\text { pacientes por } \\
\text { turno }\end{array}$ & $\begin{array}{c}\text { Horas } \\
\text { por } \\
\text { semana }\end{array}$ & $\begin{array}{c}\text { Dimensión } \\
\text { personalidad y } \\
\text { afrontamiento }\end{array}$ \\
\hline \multirow[t]{2}{*}{$\begin{array}{l}\text { Edad en } \\
\text { años }\end{array}$} & $\begin{array}{c}\text { Correlación } \\
\text { Pearson }\end{array}$ & 1 & & & & & \\
\hline & $\begin{array}{c}\text { Significancia } \\
\text { unilateral }\end{array}$ & & & & & & \\
\hline \multirow[t]{2}{*}{$\begin{array}{c}\text { Años de } \\
\text { antigüedad }\end{array}$} & $\begin{array}{c}\text { Correlación } \\
\text { Pearson }\end{array}$ & $.666^{* *}$ & 1 & & & & \\
\hline & $\begin{array}{c}\text { Significancia } \\
\text { unilateral }\end{array}$ & .000 & & & & & \\
\hline \multirow[t]{2}{*}{$\begin{array}{l}\text { Tiempo en el } \\
\text { servicio }\end{array}$} & $\begin{array}{c}\text { Correlación } \\
\text { Pearson }\end{array}$ & $.224^{* *}$ & $.204^{* *}$ & 1 & & & \\
\hline & $\begin{array}{c}\text { Significancia } \\
\text { unilateral }\end{array}$ & .002 & .005 & & & & \\
\hline \multirow[t]{2}{*}{$\begin{array}{c}\text { Número de } \\
\text { pacientes } \\
\text { por turno }\end{array}$} & $\begin{array}{c}\text { Correlación } \\
\text { Pearson }\end{array}$ & .079 & .122 & .060 & 1 & & \\
\hline & $\begin{array}{c}\text { Significancia } \\
\text { unilateral }\end{array}$ & .158 & .061 & .224 & & & \\
\hline \multirow[t]{2}{*}{$\begin{array}{c}\text { Horas por } \\
\text { semana }\end{array}$} & $\begin{array}{c}\text { Correlación } \\
\text { Pearson }\end{array}$ & $-.180^{*}$ & -.098 & $-.164^{*}$ & -.020 & 1 & \\
\hline & $\begin{array}{c}\text { Significancia } \\
\text { unilateral }\end{array}$ & .011 & .108 & .019 & .399 & & \\
\hline \multirow{2}{*}{$\begin{array}{l}\text { Dimensión } \\
\text { personalidad } \\
\text { y afronta- } \\
\text { miento }\end{array}$} & $\begin{array}{c}\text { Correlación } \\
\text { Pearson }\end{array}$ & -.016 & -.085 & .072 & .006 & $-.210^{* *}$ & 1 \\
\hline & $\begin{array}{c}\text { Significancia } \\
\text { unilateral }\end{array}$ & .422 & .142 & .182 & .471 & .004 & \\
\hline
\end{tabular}

${ }^{* *}$ Correlación significativa en un nivel 0.01 (unilateral)

Para finalizar el análisis, se buscó dependencia entre el desgaste profesional global de enfermería con nivel de estudios, servicio en el que labora y turno, los dos últimos aspectos no mostraron dependencia con el desgaste, sólo el nivel de estudios ( $X 2=10.030, p=.04$ ), en donde se destaca que, de las 25 enfermeras que presentaron nivel bajo de desgaste, 17 correspondieron a las que contaban con estudios de licenciatura, lo que refleja que el desgaste profesional depende de los años de estudio. 


\section{Discusión}

En la muestra estudiada predominó el sexo femenino en un $90.1 \%$, lo cual nuevamente evidencia que la profesión de enfermería se constituye en su mayoría por mujeres. En la literatura se encontraron datos similares, la prevalencia del sexo femenino en los estudios osciló entre $77.52 \%$ y el $91.7 \% .1,2,16,18$ ValdiviescoAmador y cols. en 2016 reportaron que la variable sexo no se asocia con el Desgaste Profesional $(p=0.62)$, resultado similar al de esta investigación $(p=0.25) .{ }^{16}$ Cabe señalar que en diversos artículos refieren que el hecho de ser mujer predispone a la aparición de este desgaste, por el doble rol que desempeñan, mujer trabajadora y madre de familia, que favorece la sobrecarga de trabajo y el aumento de los niveles de estrés, ansiedad y depresión. ${ }^{2}$, 7,16 La prevalencia de Desgaste Profesional global de los participantes en esta investigación fue de $84 \%$ mayor a la reportada por Romero y cols. en 2014, que fue de $74.6 \% .^{6}$

Con respecto a la variable edad, el grupo que predominó fue entre 37 a 46 años $(68 \%)$, dato importante ya que se demostró una relación positiva baja con la dimensión afrontamiento y personalidad $(r=0.246, p=0.001)$, lo que quiere decir que, a mayor edad, mejores estrategias para afrontar el Desgaste Profesional, dicho hallazgo es similar. Ante estos datos, conviene enfocarse al personal de enfermería en edad joven ya que el constructo de la personalidad resistente ha sido propuesto como un patrón de actitudes y habilidades que ayudan a afrontar las circunstancias estresantes en oportunidades de crecimiento que se adquieren con la edad. ${ }^{16,17}$

En cuanto al número de horas trabajadas a la semana, se encontró una correlación positiva baja con la dimensión de consecuencias $(r=.210, p=.007)$. Este resultado es consistente con otros estudios que afirman que los horarios de trabajo excesivos y extendidos son un factor de riesgo para presentar desgaste profesional $(p=.004)$, teniendo consecuencias físicas, psíquicas y organizacionales que inciden en la calidad de atención a los pacientes y en la alta tasa de ausentismo injustificado. ${ }^{2,6}$

Por otro lado, en esta investigación, la elevada carga de trabajo y el nivel de estudios del personal están entre las principales variables generadoras de Desgaste Profesional $(X 2=10.030, p=0.04)$; es decir, a menor grado académico, mayor desgaste profesional, dato importante ya que de las 25 enfermeras que presentaron nivel bajo de desgaste, ${ }^{17}$ correspondieron a las que contaban con estudios de licenciatura, resultado similar a otras investigaciones que mencionan que un factor predisponente a la aparición para este desgaste es la falta de apoyo a la formación o preparación del personal de enfermería para hacer frente a las exigencias laborales de tipo cognitivas, físicas, psíquicas y emocionales. ${ }^{7,} 15,18$

En relación con el turno laboral predominó el nocturno (34.6\%), dato importante ya que varias investigaciones mencionan que este turno es un factor de riesgo para la aparición de Desgaste Profesional o Síndrome de Burnout, por su mayor periodo de trabajo, disminución del nivel de alerta, alteración del ritmo del sueño-vigilia, aumentando los errores en procedimientos sencillos y generando perturbaciones nerviosas y psicosomáticas. Balseiro menciona en su estudio que en el $37.11 \%$ de las enfermeras que se encuentran en el turno nocturno, se observó alteración en su ritmo biológico del sueño provocando agotamiento en el 35.06\%, fatiga en el $46.87 \%$, cefaleas y migrañas en un $31.45 \%$ respectivamente. $^{2}$

A diferencia de Balseiro y Romero ${ }^{2,6}$, quienes señalan que en los servicios de enfermería que atienden a pacientes en estado crítico se presenta mayor estrés o desgaste profesional por el nivel de com- 
plejidad que requiere de dedicación, estabilidad psicológica y emocional, así como salud física y capacidad para el trabajo, 2,18 en esta investigación no se encontró dependencia entre el turno y el Desgaste Profesional Global en el personal de enfermería ( $p>0.05)$.

Por lo anterior, el Desgaste Profesional de Enfermería, se considera un trastorno adaptativo crónico a las demandas psicosociales de trabajar directamente con personas y su entorno, originando desajuste en la homeostasis biopsicosocial en donde los aspectos sociodemográficos y socio laborales son de gran importancia para la aparición de este padecimiento. ${ }^{2}$ Situación que se reporta en la presente investigación en donde las variables sociodemográficas y socio laborales como la edad, antigüedad, horas laboradas y nivel académico tienen una relación estadística significativa con los niveles de Desgaste Profesional ( $p<0.05, p<0.01$ ) resultados semejantes a otras investigaciones en donde existe una correlación positiva moderada entre las variables sociodemográficas y laborales con el índice general de burnout $(r=0.35 ; p=0.005),(r=0.35$; $p=0.004),(r=0.37 ; p=0.004)$, afectando considerablemente aspectos personales y organizacionales. ${ }^{1,5,6,15,16}$

Estos resultados constituyen un Ilamado de alerta sobre la salud del personal de enfermería de la institución donde se desarrolló la investigación, teniendo en cuenta que dicho personal ocupa hasta el $60 \%$ del total de trabajadores, por lo que se le considera la columna vertebral de estas instituciones. ${ }^{2}$

\section{Conclusiones}

La prevalencia del Desgaste Profesional de Enfermería se presenta en más de las dos terceras partes del personal estudiado, por arriba de la media nacional y se encuentra relacionado con la edad, antigüedad, nivel de estudios y horas de trabajo, lo que genera alteraciones de tipo físico, psíquico, social, familiar y organizacional.

Enfermería es una población predominantemente femenina, por lo cual es de suma importancia que la administración del hospital en el que se realizó el estudio lleve a cabo programas de prevención y estrategias de intervención a través del sistema general de riesgos laborales con la finalidad de reducir padecimientos como el DPE y el estrés.

Intervenciones de Mindfulness (Técnica basada en atención plena) mejorarían la sobrecarga de trabajo, el clima laboral, el afrontamiento como lo han implementado en estudios previos.

Aunado a lo anterior, dar una verdadera importancia a la necesidad de tener tiempo y actividades de esparcimiento que permitan fortalecer las relaciones interpersonales y estilos saludables que se conviertan en factores protectores. 


\section{Bibliografía}

1. Portero $S$, Vaquero M. Desgaste profesional, estrés y satisfacción laboral del personal de enfermería en un hospital universitario. 2015; 23: 1-10.

2. Balseiro AL. El Síndrome de Burnout como factor de riesgo laboral en el personal de enfermería. 1 ed. México: Trillas; 2010.

3. Domínguez $\mathrm{P}$, Arroyo S, Contreras M. Estado del arte del absentismo laboral en enfermería. 2014; 10(4): 5-34.

4. Llanque N. Rasgos de personalidad y síndrome de burnout en personal de Salud de la Unidad de quemados y cirugía plástica del "hospital de clínicas" de la ciudad de la paz. 2014; 12: 93-115.

5. Díaz F, Gómez I. La investigación sobre el síndrome de burnout en latinoamérica entre 2000 y el 2010. 2016; 33: 113-131.

6. Romero G, Muñoz H, Osorio $Y$, Robles D. Síndrome de Burnout en el personal de enfermería de un hospital de referencia Ibagué, Colombia, 2014 (www. um.es/eglobal/) Revista Mexicana de Enfermería. Disponible en: http://www.incmnsz.mx. Enero 2016.

7. Chacón M. Grau J. Lence J. Factores psicosociales de riesgo en profesionales asistenciales de hospitales de oncología y nivel de estrés laboral. 2014; 11: 1-36.

8. Moreno B. Gorrosa E. González J. El desgaste profesional de enfermería, desarrollo y validación factorial del CDPE. Arch. Prevención de riesgos laborales. 2000; 3:18-28

9. Ortega M. Ortiz G. Martínez A. Burnout. Burnout en estudiantes de pregrado de medicina y su relación con variables de personalidad. Terapia Psicológica. 2014; 32:235-242.

10. Ortega M. Ortiz G. Martínez A. Personalidad resistente, locus de control, autoeficacia y síndrome de burnout en estudiantes de medicina de pregrado. Universidad veracruzana.2015; 131-144.
11. Grisales H. Muñoz Y. Osorio D. et al. Síndrome de burnout en el personal de enfermería de un hospital de referencia Ibagué, Colombia, 2014. Enfermería global.2016; 41:224-257.

12. Leal $D$. Jarque D. Rehabilitar en psiquiatría ¿quema al personal de enfermería? Enfermería Global. 2014; 36: 156-167.

13. Moreno B. Gorrosa E. González G. Evaluación específica de los procesos de burnout: burnout profesorado y de enfermería. Revista latinoamericana de psicología ocupacional. 2010; 20:36-54.

14. Diario Oficial de la Federación. Reglamento de la ley general de salud en materia de Investigación para la salud, 1986, Disponible en http://www.salud.gob. $\mathrm{mx} /$ unidades/cdi/nom/compi/rlgsmis/html.

15. Fernández S. Juárez G. Arias G. Agotamiento profesional en personal de enfermería su relación con variables demográficas y laborales. Rev Enfem Inst Mex Seguro Soc. 2010; 18: 15-21.

16. Valdivieso J, Amador F, Hernández L, Hernández D. Síndrome de burnout en enfermeros que laboran en las unidades de cuidados intensivos, intermedios y Coronarios del Hospital "Joaquín Albarran".2016; 17:3-11.

17. Carmona $\mathrm{H}$, Garrosa $\mathrm{E}$, Moreno B. Análisis psicométrico de la escala de personalidad resistente (EPR) adaptada a estudiantes universitarios chilenos. 2017; 42: 286-292.

18. Blanco L, García C, Moreno B, Pinta M, Moraleda $S$, Garrosa E. Intervención breve basada en ACT y mindfulness: estudio piloto con profesionales de enfermería en $\mathrm{UCl}$ y Urgencias. International Journal of Psychology and Psichological Therapy. 2017; 17(1):57-73.

19. Gorrosa E, Moreno B. Salud Laboral "riesgos laborales psicosociales y bienestar Laboral". 1 ed. Madrid: PIRAMIDE; 2014

20. Flaxman P. Blackledg J. Bond F. Acceptance and commitment therapy. 1 ed. London and New York: Routledge. 2011 